\title{
ÉTICA E PROMOÇÃO DA SAÚDE SOB A ÓTICA DE ENFERMEIROS DA ATENÇÃO BÁSICA
}

\author{
ETHICS AND HEALTH PROMOTION FROM THE \\ PERSPECTIVE OF PRIMARY CARE NURSES
}

\section{ÉTICA Y PROMOCIÓN DE LA SALUD DESDE LA PERSPECTIVA DE LOS ENFERMEROS DE ATENCIÓN PRIMARIA}

\author{
Kátia Pereira de Borba ${ }^{1}$ \\ Donizete Azevedo dos Santos Silva ${ }^{2}$ \\ Leonardo de Carvalho Barbosa Santos ${ }^{3}$ \\ Isabela Leticia Petry ${ }^{4}$ \\ Maria José Clapis ${ }^{5}$ \\ Adriana Katia Corrêa ${ }^{6}$
}

Como citar este artigo: Borba KP, Silva DAS, Santos LCB, Petry IL, Clapis MJ, Corrêa AK. Ética e promoção da saúde sob a ótica de enfermeiros da Atenção Básica. Rev baiana enferm. 2021;35:e43116.

Objetivo: identificar a percepção de enfermeiros da Atenção Básica sobre a ética na realização de ações de promoção da saúde. Método: estudo descritivo exploratório de natureza qualitativa, realizado com 14 enfermeiros atuantes em unidades de Estratégia Saúde da Família. Os dados obtidos por entrevista semiestruturada foram analisados mediante a análise de conteúdo na modalidade temática. Resultados: evidenciaram-se conflitos de ética profissional entre enfermeiros e médicos; relação de vínculo entre usuários e enfermeiros; condutas de promoção da saúde pelo enfermeiro com enfoque biologicista; justificativa de assistência inadequada aos usuários, por motivo de sobrecarga de trabalho e equipe de enfermagem incompleta; sofrimento moral pelo enfermeiro durante a experiência profissional. Considerações finais: na perspectiva de enfermeiros atuantes na Atenção Básica, a ética na realização de ações de promoção da saúde está relacionada à diversidade de trabalho cotidiano decorrente da experiência profissional.

Descritores: Ética Profissional. Ética em Enfermagem. Enfermagem em Saúde Comunitária.

Objective: to identify the perception of primary care nurses about ethics in health promotion actions. Method: exploratory descriptive study of qualitative nature, carried out with 14 nurses working in Family Health Strategy units. The data obtained from semi-structured interview were analyzed through content analysis in the thematic modality. Results: conflicts of professional ethics between nurses and physicians were evidenced, in addition to the relationship between users and nurses; health promotion behaviors by nurses with a biologicist approach; justification of inadequate care to users, due to work overload and incomplete nursing staff; nurses' distress during professional experience. Final considerations: according to the nurses working in Primary Care, ethics in health promotion actions is related to the diversity of daily work resulting from professional experience.

Descriptors: Professional Ethics. Ethics in Nursing. Community Health Nursing.

\footnotetext{
Enfermeira. Professora da Universidade Estadual do Centro-Oeste. Guarapuava, Paraná, Brasil. kborba@unicentro.br. http://orcid.org/0000-0003-2I64-4289. Estudante de Enfermagem. Universidade Estadual do Centro-Oeste. Guarapuava, Paraná, Brasil. http://orcid.org/0000-000 I-7586- I 065.

Estudante de Enfermagem. Universidade Estadual do Centro-Oeste. Guarapuava, Paraná, Brasil. http://orcid.org/0000-0002-4869-342X

Estudante de Enfermagem. Universidade Estadual do Centro-Oeste. Guarapuava, Paraná, Brasil. http://orcid.org/0000-0002-6593-4686.

Enfermeira. Professora visitante da Universidade Federal de Alfenas. Alfenas, Minas Gerais, Brasil. http://orcid.org/0000-0002-6593-4686.

Enfermeira. Professora da Universidade de São Paulo. Ribeirão Preto, São Paulo, Brasil. http://orcid.org/0000-0003-| $496-6108$.
} 
Objetivo: identificar la percepción de los enfermeros de atención primaria sobre la ética en las acciones de promoción de la salud. Método: estudio descriptivo exploratorio de carácter cualitativo, realizado con 14 enfermeros que trabajan en unidades de Estrategia de Salud Familiar. Los datos obtenidos por entrevista semiestructurada fueron analizados a través del análisis de contenido en la modalidad temática. Resultados: se evidenciaron conflictos de ética profesional entre enfermeros y médicos; relación entre usuarios y enfermeros; la promoción de la salud realizada por enfermeros con enfoque biológico; justificación de la atención inadecuada a los usuarios, debido a la sobrecarga de trabajo y al personal de enfermería incompleto; sufrimiento de los enfermeros durante la experiencia profesional. Consideraciones finales: desde la perspectiva de los enfermeros que trabajan en Atención Primaria, la ética en las acciones de promoción de la salud está relacionada con la diversidad del trabajo diario resultante de la experiencia profesional.

Descriptores: Ética Profesional. Ética en Enfermería. Enfermería de Salud Comunitaria.

\section{Introdução}

Ética é a ciência que investiga os atos humanos, acarretando comportamentos que envolvem valores e princípios ${ }^{(1)}$. Valores e princípios éticos amparam-se em teorias filosóficas que se classificam em: empírica, que envolve a experiência; pura, que é sustentada por princípios que antecedem a experiência; e metafísica, filosofia pura, limitada a determinadas áreas do conhecimento $^{(2)}$.

A metafísica compreende a natureza do indivíduo, na sua forma física e em seus costumes. Por isso, é indispensavelmente necessária, não só por motivos de ordem especulativa, para investigar a fonte dos princípios práticos que antecedem a razão do indivíduo, mas também porque os próprios costumes ficam sujeitos à desonestidade na falta do autojulgamento ${ }^{(2)}$.

Diante dessas considerações teóricas, compreende-se a importância da ética enquanto elemento transversal nas circunstâncias factuais que envolvem o processo de trabalho de enfermeiros que têm a Atenção Básica à Saúde (ABS) como campo de atuação. Neste campo de trabalho, as atividades de cuidar e assistir compreendem ações de acompanhamento contínuo do indivíduo, da família e da comunidade, objetivando a prevenção de doenças e a recuperação e promoção da saúde (PS) ${ }^{(3)}$.

A PS é um processo que se caracteriza pela autorresponsabilização do indivíduo pela própria saúde. O objetivo primordial é amenizar divergências na saúde da população e garantir benefícios e recursos igualitários que potencializem um adequado estado de saúde para todas as pessoas. A PS envolve ambientes favoráveis, acesso à informação, experiências e habilidades na vida, além de oportunidades que permitam ao indivíduo fazer escolhas voltadas a uma vida mais saudável. Sobretudo, é um processo que depende de uma ação coordenada entre governo e setores sociais, econômicos e de saúde, assim como organizações não-governamentais, autoridades locais, indústria e mídia ${ }^{(4)}$.

Visando a PS, a ABS concretiza, na Estratégia Saúde da Família (ESF), o desenvolvimento do trabalho de cuidar da saúde de indivíduos, da família e da comunidade. A ESF tem o objetivo de consolidar os princípios estabelecidos pelo sistema público de saúde brasileiro, o Sistema Único de Saúde (SUS), e responder às necessidades de saúde da população ${ }^{(5)}$.

As ações de PS realizadas na ESF deverão ser conduzidas pelas diretrizes da Política Nacional de Promoção à Saúde (PNPS). Instituída na gestão do SUS, esta política considera o enfermeiro protagonista na execução das atividades promotoras da saúde, por ser um profissional que estimula e cria vínculo com a equipe de saúde interdisciplinar e a comunidade ${ }^{(3)}$.

A fim de desenvolver ações de PS na ABS, o enfermeiro deve utilizar estratégias que impulsionem modificações no setor saúde e na sociedade, possibilitando a recuperação de valores essenciais para a construção de novas relações sociais pautadas no respeito, na solidariedade e na ética ${ }^{(5)}$. Em se tratando da ética, ressalta-se a 
possibilidade do confronto de crenças e valores que o processo de proximidade entre o enfermeiro e as distintas formas de viver de indivíduos da comunidade assistida possa promover.

Partindo da premissa que as ações de PS realizadas por enfermeiros da ABS envolvem questões éticas entre indivíduos, família, comunidades e profissionais de saúde, surgiu o interesse pela realização deste estudo, com a seguinte questão de pesquisa: Como enfermeiros atuantes na ABS percebem a ética no desenvolvimento de ações de PS?

O objetivo deste estudo foi identificar a percepção de enfermeiros da Atenção Básica sobre a ética na realização de ações de promoção da saúde.

\section{Método}

Pesquisa descritiva exploratória de natureza qualitativa, realizada junto à Secretaria Municipal de Saúde (SMS) de um município do Sul do Brasil, com enfermeiros atuantes em unidades de ESF. Os enfermeiros investigados foram identificados em uma lista fornecida pelo setor de educação permanente da SMS do município cenário deste estudo.

A inclusão dos participantes no estudo caracterizou-se por amostragem qualitativa intencional $^{(6)}$, construída com base nos 32 critérios consolidados para relatar pesquisa qualitativa - Consolidated criteria for reporting qualitative (COREQ). Em observância ao guia internacional COREQ, participaram do estudo os enfermeiros que compartilhavam características particulares e com potencial de fornecer dados ricos, relevantes e diversos, pertinentes à questão de pesquisa ${ }^{(6)}$. Critério de inclusão: enfermeiros atuantes na ESF por um período mínimo de um ano. Critério de exclusão: enfermeiros que, no período programado para coleta de dados, estavam ausentes do serviço no momento da realização da entrevista.

O período entre os meses de setembro e dezembro de 2019 foi destinado à coleta de dados. Para isto, foi utilizado um instrumento de entrevista semiestruturada contendo perguntas abertas e fechadas sobre a caracterização dos sujeitos, quanto a sexo, idade, tempo de profissão e atuação na ESF; e questões norteadoras que envolveram a ética e as ações de PS.

As entrevistas, realizadas pelos próprios pesquisadores nas salas individuais de trabalho dos enfermeiros, de acordo com a disponibilidade deles, foram gravadas, com duração mínima de 15 e máxima de 60 minutos. Estavam presentes no momento somente o pesquisador e o pesquisado. Primeiramente, realizou-se um teste piloto, o que envolveu três entrevistas. Como não se considerou haver necessidade de modificações das questões, essas entrevistas foram agregadas à amostragem qualitativa total. Contudo, não foi necessária a repetição de entrevistas. Cada investigado foi identificado pela letra E (Enfermeiro) seguida do número correspondente à ordem de realização da entrevista.

Analisaram-se os dados obtidos mediante a técnica de análise de conteúdo, na modalidade temática $^{(6)}$, compreendendo as seguintes etapas: pré-análise - de forma exaustiva, realizou-se a leitura do material selecionado, objetivando a compreensão textual e uma visão de conjunto; tratamento dos resultados - distribuição dos textos de análise em trechos, frases e fragmentos de falas, identificando-se, por meio de inferências, os núcleos de sentido; mediante a categorização dos dados, foram definidas duas categorias de análise; elaboração da síntese interpretativa - construção da redação por tema, com o propósito de articular e dar sentido aos textos que orientaram a análise.

Este estudo seguiu os preceitos da Resolução $n^{\circ} 466 / 2012$ do Conselho Nacional de Saúde. $\mathrm{O}$ projeto de pesquisa foi aprovado pelo Comitê de Ética em Pesquisa da Universidade Estadual do Centro-Oeste do Paraná, sob o Parecer $\mathrm{n}^{\mathrm{o}} 3.527 .560$.

\section{Resultados}

Participaram do estudo 11 enfermeiras e 3 enfermeiros. Dos 14 enfermeiros participantes, 8 tinham idade entre 30 e 40 anos; 3 , entre 41 e 50 anos; e 3, mais de 60 anos. Em relação ao tempo de profissão, 7 enfermeiros tinham entre 
5 e 10 anos; 3, entre 11 e 20 anos; e 4, mais de 20 anos. Um atuava entre 1 e 2 anos na ESF; 6, entre 5 e 10 anos; e 7, mais de 10 anos.

Os resultados das entrevistas semiestruturadas estão dispostos em duas categorias de análise - Ética e promoção da saúde no contexto da organização da Estratégia Saúde da Família e Questões éticas nas ações de Promoção da Saúde envolvendo enfermeiros e pessoas usuárias do SUS -, cada uma organizada em diferentes subcategorias.

\section{Ética e promoção da saúde no contexto da organização da Estratégia Saúde da Família}

Fazem parte desta categoria, as subcategorias indicadas a seguir.

\section{Elementos envolvendo a ética profissional entre a equipe interdisciplinar da ESF}

\begin{abstract}
A questão ética nas ações de PS [Promoção da Saúde] envolve a equipe multiprofissional, ou seja, há resistência dos profissionais de saúde para querer mudar o modelo biomédico de atenção à saúde. Ainda tem muitos profissionais de saúde que acreditam que, para manter a saúde, é só tomar remédio. Isso gera conflito ético, porque influencia os usuários. (E2).
\end{abstract}

No caso da equipe interdisciplinar, envolve a questão do sigilo sobre aquilo que é discutido nas reuniões. Gera conflito moral, quando esse sigilo é quebrado por algum membro da equipe. (E3).

Pessoas que se queixam de alguns profissionais, como o médico, por exemplo, e, muitas vezes, ficamos de mãos atadas. (E11).

Sobrecarga de trabalho e equipe de enfermagem incompleta

Se tivesse uma maior cobertura do ESF [Estratégia Saúde da Família], poderíamos trabalhar melhor a $P S$ [Promoção da Saúde] e não ficariamos somente apagando fogo. (E2).

Sobrecarga. É muito complicado. Não se trabalha tranquilo, mas sob pressão o tempo todo. Faço o possível para realizar ações coletivas de PS [Promoção da Saúde]. (E11).

\section{Conflito moral entre enfermeiro e médico}

Muitas vezes, nós, que estamos no acolbimento, percebemos que o usuário não precisa passar pelo médico e entendemos que podemos resolver, somente conversando com o paciente, mas estamos ali para organizar a fila do atendimento, classificar o risco. (E2).

Penso no fato de as pessoas preferirem o atendimento médico. Às vezes, a ESF [Estratégia Saúde da Família] fica três meses sem ter dentista e não tem qualquer reclamação. Agora, se a ESF fica uma semana sem médico, é complicado! É cultural o atendimento centrado no médico. (E5).

Recentemente, eu tive uma situação desgastante. Um médico falou para mim que eu não podia questioná-lo quanto ao diagnóstico do paciente, mesmo que eu não concordasse com sua avaliação médica. (E6).

Tenho problemas com o profissional médico. Ele reclama de atender os pacientes, é negligente nos atendimentos, sejam de diagnóstico ou procedimentais, e isso, para mim, é muito difícil, porque as pessoas que vêm aqui, esperam muito da gente. (E12).

\section{Atendimentos individuais e de consultas de enfermagem configurando ações de Promoção da Saúde}

Dizer à gestante, a mãe, no momento da puericultura, sobre os cuidados para prevenir doenças e evitar problemas. (E3).

Às vezes, até com uma simples conversa no balcão, a gente faz muito essa PS [Promoção da Saúde], ou seja, ao entregar a medicação, orientando a pessoa sobre como tomar, também fazendo orientações no momento da pré-consulta. (E5).

As minhas ações de PS [Promoção da Saúde] são em relação às consultas agendadas, às orientações no salão, quando eles estão esperando a consulta médica. Também faço orientações no acolbimento e classificação de risco. (E6).

É estar sempre orientando. É o acolbimento, a escuta do paciente. Ter uma escuta ativa. (E7).

Eu aproveito as consultas de enfermagem para fazer PS [Promoção da Saúde]. (E4).

\section{Intenção da formação de vínculo entre} enfermeiros e a população adscrita, e desenvolvimento da autonomia do usuário no cuidado em saúde

É incentivar o indivíduo a fazer atividades físicas, ter uma alimentação adequada, ao autocuidado. (E7).

Temos investido fortemente num projeto de borta comunitária. A ideia é envolver nossa comunidade para o autocuidado. (E2).

Preocupar-se com a pessoa e não com a doença. (E10).

Eu acho que é qualquer tipo de ação que a gente faça, e que leva a pessoa a entender que ela também é responsável pela sua saúde. (E11) 
Levar o conbecimento para o usuário e que, a partir desse conbecimento, ele passe a se cuidar. (E12).

Estimular o autocuidado. Despertar no usuário a consciência, por exemplo, da alimentação adequada e da realização anual do preventivo do câncer ginecológico. (E14).

\section{Questões éticas nas ações de Promoção da Saúde envolvendo enfermeiros e pessoas usuárias do Sistema Único de Saúde}

Fragmentos de dados relativos a esta categoria estão expostos nas subcategorias a seguir.

\author{
Cumprimento da ética profissional com \\ respeito e sigilo no atendimento ao usuário
}

Quando você faz um atendimento de enfermagem, seja para qualquer pessoa, independente de credo, religião, o importante é o respeito; ou seja, o paciente deve ser respeitado, ouvido e sua integridade deve ser preservada. (E5).

A ética no atendimento envolve o respeito, o sigilo. Por exemplo, os relatos que eu ouço no momento da coleta de um Papanicolau devem dizer respeito somente a mim e à paciente. (E6).

A ética envolve um atendimento com respeito; ou seja, não expor o paciente, para não o constranger; não usar termos técnicos, porque "eles" não entendem; não ferir a confiança do paciente; não fazer críticas à queixa do paciente. (E7).

Eu já tive situações de orientar o paciente sobre uma alimentação correta, como diminuir o consumo de gordura, por exemplo, e ele não acatar, porque é da cultura dele comer de manhã cedo virado de feijão com ovo frito e torresmo. Outra situação é a gestante, em que você orienta a importância de fazer atividade física, ingerir bastante liquido para evitar infecção urinária e, na sua frente, elas concordam, mas nem sempre fazem o que você orientou. E o que você faz com tudo isso? Orienta, mas se o paciente não acatar, respeita. (E13).

\section{Sofrimento moral do enfermeiro decorrente} de sua experiência profissional na Estratégia Saúde da Família

Quando se tem uma situação de abuso sexual e na tomada de decisão para denunciar, você fica coagido pela situação de trabalhar naquela ESF [Estratégia Saúde da Família] e ser conbecido no território. (E10).

Aqui tem muitos casos de mulheres que vivem em situação de violência, que é assustador, sabe? (E12).

Remédios que as pessoas precisam e não conseguem, porque dependem de licitação; e uma hora outra falta, e a pessoa tem dependência e fica sem. Isso me abala! (E11).

Uma situação é o fato de o usuário ser resistente para ouvir o profissional de saúde, sendo a justificativa: Minha mãe fazia assim e eu vou continuar a fazer assim. Isso, para mim, é um dilema ético. Eu sofro com isso! (E2).

Uma questão ética é quando atendemos uma paciente $H I V$ [vírus da imunodeficiência humana] positiva, que já estava no terceiro filho, e o parceiro atual não sabia do diagnóstico, sendo que ela não queria contar para ele. Entendo essa situação como um dilema ético, que me impõe um sofrimento moral. Como eu posso conviver com isso, sabendo que o parceiro dela estava correndo risco, $e$ eu, enquanto profissional de saúde, estava de mãos atadas? Se eu quebrasse o sigilo, ou não, eu parecia não estar cumprindo com a minha ética profissional. Outra situação é a criança com suspeita de abuso sexual. A gente acaba tentando defender a criança, mas aí o adulto, às vezes, não aceita, acha que não é, ou protege o abusador. Sofro muito com tudo isso! (E3).

Então, eu acompanbei uma gestante com 13 anos, casada, que engravidou porque parou de usar anticoncepcional por vontade própria; ou seja, foi uma gestação planejada. Para mim, isso é um dilema ético, porque eu tive que atender a gestante sem julgá-la pela gravidez, que eu entendo que não era o momento ideal para essa menina de 13 anos ter um filbo! (E10).

Sofro com dilemas que esbarram naquilo que eu acredito como correto como, ao atender uma criança em situação de abuso sexual, em que você percebe que o autor do crime é um parente bem próximo, como o avô, por exemplo. Uma outra situação seria a de uma mulber pedindo ajuda para fazer um aborto, sendo que os meus princípios morais e valores não aceitam, independente do motivo. (E14).

\section{Discussão}

Questões éticas integrantes das atividades cotidianas desenvolvidas pelo enfermeiro na ESF, quando não percebidas e valorizadas, podem resultar em danos na atenção à saúde da população adscrita, principalmente no que diz respeito a vínculo e corresponsabilidade ${ }^{(8)}$. Nessa perspectiva, enfermeiros que atuam na ESF, além de exercerem adequadamente o trabalho de cuidar da saúde da população, especialmente realizando ações de PS, precisam ter a sensibilidade para perceber e considerar questões éticas de caráter significativo. Assim, considera-se que a relação profissional entre equipe e usuário fica estremecida, quando são promovidas pelo confronto de crenças, valores e diferentes formas de viver.

Desempenhar o trabalho de PS na ESF exige do enfermeiro situar-se no paradigma da gestão de redes e ter uma visão generalista, o que abarca conhecimentos na área da política, da educação, da cultura, dos esportes, da habitação, do meio ambiente e/ou qualquer um outro que tenha confluência com o campo da saúde coletiva ${ }^{(9)}$. 
Implica ainda na efetivação de um processo de trabalho marcado pelo exercício da cidadania, isto é, na oferta de uma prática de cuidado com qualidade de relação interpessoal entre profissional e usuário, permeada por questões éticas que exigem valorização e não se restringem ao atendimento clínico ${ }^{(8)}$.

Neste estudo, a PS não foi relacionada às condições de moradia, cultura e educação da população adscrita, assim como a responsabilidade social e política envolvida. Sobretudo, os enfermeiros apresentaram um discurso amplo sobre o dinamismo saúde-doença, sobrepujando um conhecimento simplificado de saúde.

Entre os enfermeiros investigados, a compreensão sobre PS não se demonstrou qualificada, ficando implícitas dificuldades e incertezas sobre esse conceito ampliado de saúde. Corrobora esses achados, estudo realizado com enfermeiros da ESF do município de São Carlos (SP), no qual dificuldades em demonstrar conhecimento sobre PS foram evidências significativas entre os profissionais ${ }^{(10)}$.

O entendimento sobre PS no contexto da ESF deve estar inserido nas condutas de saúde de domínio individual e coletivo, o que envolve uma atenção integral que repercuta nos determinantes sociais de saúde de indivíduos e comunidades ${ }^{(5)}$. Esse entendimento pode ser disposto em dois grandes eixos: um aproxima as ações de promoção das de prevenção de doenças; o outro, propõe a ampliação do conceito de saúde, o que envolve a preocupação das próprias pessoas pela edificação de melhores condições de vida ${ }^{(9)}$.

Ressalta-se que a concepção de prevenção é norteada por conceitos biomédicos, que têm a saúde como ausência de doenças ${ }^{(11)}$. Contudo, a prevenção de doenças é um termo que está inserido na PS, visto que ações de PS previnem doenças $^{(9)}$. Talvez essa aproximação entre o conceito de PS e a prevenção de doenças seja explicada pelo fato de os enfermeiros deste estudo atribuírem às ações de PS um caráter biologicista.

As ações de PS realizadas na ESF devem ser conduzidas segundo as diretrizes da PNPS. Destaca-se, nos relatos dos enfermeiros, a naturalização de considerarem que os atendimentos individuais e as consultas de enfermagem configuram ações de PS, caracterizando um distanciamento das diretrizes da PNPS. Outro fator relevante foi a sobrecarga de trabalho e a equipe de enfermagem incompleta servirem de justificativa para os enfermeiros não realizarem ações coletivas de PS. Relaciona-se esses achados ao estudo ${ }^{(10)}$ no qual os enfermeiros também relataram desenvolver, de forma predominante, ações de PS nos atendimentos individuais e de consultas de enfermagem.

As consultas de enfermagem são imprescindíveis à atuação do enfermeiro, a fim de identificar as necessidades do usuário, assim como fortalecer o vínculo com ele. Contudo, as atividades promotoras da saúde não devem limitar-se à assistência individual, mas enfatizarem as estratégias para a realização de condutas coletivas de PS, como o ato de educar em saúde ${ }^{(10)}$.

A educação em saúde está estreitamente associada ao cuidado prestado às pessoas, assim como conduz diferentes ações exercidas pelos enfermeiros, os quais são educadores por excelência. As ações educativas caracterizam-se como um dos mais importantes elementos para o desenvolvimento de ações de $\mathrm{PS}^{(10-11)}$. Nesse tipo de estratégia, devem ser abordados temas que englobem os determinantes sociais que interferem no processo de saúde-doença, a exemplo da mobilidade segura e sustentável e também das práticas corporais saudáveis ${ }^{(3)}$.

Embora as ações de PS relatadas pelos enfermeiros fossem de caráter individual e com enfoque na prevenção, verificou-se a intenção da equipe interdisciplinar da ESF de formar vínculo com a população adscrita, além do desenvolvimento da autonomia do usuário no cuidado em saúde. Relaciona-se esses achados a um estudo $^{(10)}$ realizado em Recife (PE), com o objetivo de analisar a percepção do enfermeiro sobre sua atuação educativa na ESF, e outro desenvolvido no município de São Carlos (SP), que analisou o conhecimento e a prática de promoção de saúde realizada por enfermeiros de $\mathrm{ESF}^{(12)}$. O sucesso de ações coletivas de PS está relacionado à motivação e ao envolvimento da equipe organizadora, além de abranger o sentimento de 
pertencimento dos participantes dos grupos e o reconhecimento de seus saberes e de suas necessidades de saúde ${ }^{(13)}$.

Diante desse contexto, entende-se que a ética nas ações de PS aglutina-se em uma diversidade de ações em saúde, apoiadas em métodos, técnicas e procedimentos que inspiram o desenvolvimento do trabalho do enfermeiro no enfrentamento de inúmeros problemas de saúde que afetam os indivíduos, as famílias e a comunidade. Em especial na prática cotidiana do enfermeiro, o cuidado deve constituir a essência do seu fazer, isto é, ir além da prática biologicista e fragmentada ${ }^{(14)}$.

A relação interprofissional entre enfermeiros e médicos demonstrou-se conturbada, sendo pano de fundo a competência da formação acadêmica. Corroboram esses achados os estudos realizados com enfermeiros da $\operatorname{APS}^{(15)}$ e enfermeiros da $\mathrm{ESF}^{(16)}$. Destarte, o conflito moral entre enfermeiro e médico, envolvendo discrepância de autoridade, é historicamente construído. Contrário ao momento contemporâneo, isso acontecia em um tempo em que a medicina era comumente executada por homens e a enfermagem por mulheres. Todavia, a estrutura de poder de gênero entre essas categorias profissionais persiste na imaginação da sociedade atual, mesmo que em tempos contemporâneos tenha-se um número considerável de mulheres na medicina e de homens na enfermagem ${ }^{(17)}$.

Entende-se que aplicar a ética no desenvolvimento das ações de PS implica em princípios filosóficos, experiências histórico-sociais e no comportamento humano. Importante ressaltar que a ética tem a função de explicar, esclarecer e estudar as relações e o comportamento da espécie humana dentro da sociedade, a fim de elaborar conceitos que indiquem o melhor comportamento do ponto de vista moral. Por mais que a moral tenha determinados princípios, não é a ética que os esclarece. A ética, enquanto ciência da moral, depara-se com experiências histórico-sociais ${ }^{(18)}$. Uma ação moralmente boa é aquela realizada de acordo com certos princípios universais. Quem age segundo princípios não se preocupa com o resultado de suas ações. Quem se preocupa exclusivamente com o resultado de suas ações, faz aquilo que é necessário para que ocorra o que deseja ${ }^{(19)}$.
O sofrimento moral dos enfermeiros participantes deste estudo, decorrente da proximidade nos atendimentos a usuários, é confirmado em um estudo ${ }^{(20)}$ que objetivou verificar os problemas éticos vivenciados por enfermeiros atuantes numa ESF de São Paulo, o qual evidenciou que a ética, na ABS, ocupa-se de acontecimentos comuns na prática diária do trabalho do enfermeiro.

Enfermeiros, quando participam de ações percebidas como incorretas, seja por ação ou omissão, podem ser atingidos pelo sofrimento moral, que é circundado pelo sofrimento psicológico, emocional e fisiológico ${ }^{(21)}$. Estudo ${ }^{(22)}$ sobre sofrimento moral em enfermeiros atuantes em unidades de ABS no Rio Grande do Sul identificou, entre seus resultados, a contestação de decisões de outros profissionais como um fator de risco que apresentou as maiores médias. Há também os estudos realizados na Nova Zelândia $^{(23)}$ e na China ${ }^{(24)}$ que apontam índices consideráveis de sofrimento moral entre enfermeiros. Compreende-se ser necessário debruçar-se sobre as questões éticas que envolvem o cotidiano do trabalho em saúde, seja nas relações profissional-paciente, seja nas relações profissional-equipe interdisciplinar ${ }^{(25)}$.

Enfermeiros atuantes na ABS/ESF precisam incorporar a PS como uma estratégia de transformação social $^{(4)}$. É importante refletir sobre o cuidado e destacar que, na profissão Enfermeiro, cuidar é muito mais do que proteger, do que fornecer suporte psicológico e emocional, satisfazendo o indivíduo no cuidado de suas necessidades. Cuidar é ser eticamente competente, prestar um serviço com respeito, tomar decisões sábias e oportunas. Quando o enfermeiro exerce a prática do cuidar em saúde, fazendo uso de princípios éticos, o relacionamento entre "ele" e o indivíduo que é cuidado torna-se fortalecido ${ }^{(26)}$. Assim, considera-se sine qua non a sensibilidade do enfermeiro em relação às questões éticas que advém nas ações de PS junto a indivíduos, família e comunidade.

Tendo em vista a relevância dos resultados deste estudo, considerou-se o pequeno número de participantes na pesquisa uma limitação. Outra limitação considerada foi a escassez de estudos científicos que abordassem a temática 
ética nas ações de PS por enfermeiros atuantes na ABS, nessa conjuntura, fato que inibiu comparações mais valiosas.

Acredita-se que os resultados aqui apresentados possam desencadear a expansão de conhecimentos junto à temática ética e PS com foco na ABS, subsidiando, na perspectiva deontológica, a construção de práticas compromissadas no contexto do trabalho em enfermagem, solidificando a adequada assistência prestada junto aos usuários do SUS.

\section{Considerações Finais}

A realização deste estudo confirmou a premissa inicial que o motivou, identificando-se a relação entre questões éticas e ações de PS entre enfermeiros que atuam na ABS.

As ações de PS relatadas pelos enfermeiros foram de caráter individual e com enfoque na prevenção. Contudo, percebeu-se a intenção da formação de vínculo entre enfermeiros e população adscrita, e o desenvolvimento da autonomia do usuário no cuidado em saúde.

Dentre os elementos envolvendo a ética e as ações de PS apontados pelos enfermeiros destacaram-se: desconfortos de ordem profissional entre a equipe multiprofissional, desencadeados por sobrecarga de trabalho; equipe de enfermagem incompleta; e sofrimento moral. Em contrapartida, os enfermeiros demonstraram acreditar cumprir a ética profissional, ao assistir o usuário com o devido respeito e assegurar o sigilo do atendimento.

Sob a ótica de enfermeiros atuantes na ABS, a ética nas ações de PS está, sobretudo, relacionada à diversidade de trabalho cotidiano decorrente da experiência profissional.

\section{Colaborações:}

1 - concepção, projeto, análise e interpretação dos dados: Kátia Pereira de Borba, Donizete Azevedo dos Santos Silva, Leonardo de Carvalho Barbosa Santos e Isabela Letícia Petry;

2 - redação do artigo e revisão crítica relevante do conteúdo intelectual: Kátia Pereira de Borba, Maria José Clapis e Adriana Kátia Corrêa;
3 - aprovação final da versão a ser publicada: Kátia Pereira de Borba, Maria José Clapis e Adriana Katia Corrêa.

\section{Referências}

1. Burgatti JC, Bracialli LAD, Oliveira MAC. Ethical problems experienced in a supervised curricular internship in nursing in an integrated curriculum. Rev Esc Enferm USP. 2013;47(4):934-9. DOI: 10.1590/S0080-623420130000400023

2. Kant E. Fundamentação da Metafísica dos Costumes. 70a ed. Lisboa: Portugal; 2007.

3. Brasil. Ministério da Saúde. Política Nacional de Promoção da Saúde. Revisão da Portaria MS/GM n ${ }^{\circ}$ 687, de 30 de março de 2006 [Internet]. Brasília (DF); 2014 [cited 2020 Aug 20]. Available from: http://bvsms.saude.gov.br/bvs/publicacoes/ politica_nacional_promocao_saude_pnaps.pdf

4. World Health Organization. The Ottawa for health promotion. Ottawa; 1986.

5. Brasil. Ministério da Saúde. Portaria n ${ }^{\circ} 2.436$, de 21 de setembro de 2017. Aprova a Política Nacional de Atenção Básica, estabelecendo a revisão de diretrizes para a organização da Atenção Básica, no âmbito do Sistema Único de Saúde (SUS) [Internet]. Brasília (DF); 2017 [cited 2020 Aug 30]. Available from: https://bvsms.saude.gov.br/bvs/ saudelegis/gm/2017/prt2436_22_09_2017.html

6. Minayo MC, Deslandes SF, Gomes R. Pesquisa Social: teoria, método e criatividade. Petrópolis: Vozes; 2016. Série Manuais Acadêmicos.

7. Tong A, Sainsbury P, Craig J. Consolidated criteria for reporting qualitative research (COREQ): a 32-item checklist for interviews and focus groups. Int J Qual Health Care. 2007;19(6):349-57. DOI: https://doi.org/10.1093/intqhc/mzm042

8. Lima CA, Oliveira APS, Macedo BF, Dias OV, Costa SM. Relação profissional usuário de saúde da família: perspectiva da bioética contratualista. Rev bioét (Impr). 2014;22(1):152-60.

9. Czeresnia D, Freitas CMD. Promoção da Saúde: conceitos, reflexões, tendências. Rio de Janeiro: Fiocruz; 2017.

10. Silva NCC, Mekaro KS, Santos RIO, Uehara SCSA. Knowledge and health promotion practice of Family Health Strategy nurses. Rev Bras Enferm. 2020;73(5):e20190362. DOI: 10.1590/0034-7167-2019-0362 
11. Iglesias A, Dalbello-Araújo M. As concepções de promoção da saúde e suas implicações. Cad Saúde Colet [Internet]. 2011 [cited 2017 Mar 23];19(3):291-8. Available from: http:// www.cadernos.iesc.ufrj.br/cadernos/images/ csc/2011_3/artigos/csc_v19n3_291-298.pdf

12. Andrade ME, Clares JWB, Barretto EMF, Vasconcelos EMR. Nurses' perceptions of their educational role in the family health strategy. Rev enferm Uerj. 2016;24(4):e15931. DOI: http:// dx.doi.org/10.12957/reuerj.2016.15931

13. Nogueira ALG, Munari DB, Fortuna CM, Santos LF. Leads for potentializing groups in Primary Health Care. Rev Bras Enferm. 2016;69(5):964-71. DOI: https://doi.org/10.1590/ 0034-7167-2015-0102

14. Santos FPA, Acioli S, Rodrigues VP, Machado JC, Souza MS, Couto TA, et al. Nurse care practices in the Family Health Strategy. Rev Bras Enferm. 2016;69(6):1060-7. DOI: http://dx.doi. org/10.1590/0034-7167-2016-0273

15. Nora CRD, Zoboli ELCP, Vieira MM. Moral sensitivity in Primary Health Care nurses. Rev Bras Enferm. 2017;70(2):308-16. DOI: http:// dx.doi.org/10.1590/0034-7167-2016-0453

16. Dourado JCL, Aguiar FAR, Lopes REL, Silva MAM, Ferreira Júnior AR. Problemas éticos vivenciados por enfermeiros na Estratégia Saúde da Família. Rev Bioét [Internet]. 2020 [cited 2020 Jul 24];28(2):356-64. Available from: https:// www.scielo.br/pdf/bioet/v28n2/en_1983-8042bioet-28-02-0356.pdf

17. Galbany-Estragués P, Comas-D’Argemir D. Care, Autonomy, and Gender in Nursing Practice: A Historical Study of Nurses' Experiences. J Nurs Res. 2017;25(5):361-7. DOI: 10.1097 / JNR. 0000000000000184

18. Vásquez AS. Ética. Rio de Janeiro: Civilização Brasileira; 2002.
19. Bobbio N. Ética e política. Lua Nova. 1992;(25):131-40. DOI: https://doi.org/10.1590/ S0102-64451992000100006

20. Zoboli ELCP. Nurses and primary care service users: bioethics contribution to modify this professional relation. Acta paul enferm. 2007;20(3):316-20. DOI: https://doi.org/10.1590/ S0103-21002007000300012

21. Mccarthy J, Gastmans C. Moral distress: a review of the argument-based nursing ethics literature. Nurs Ethics. 2015;22(1):131-52. DOI: 10.1177 / 0969733014557139

22. Schaefer R, Zoboli ELCP, Vieira M. Moral distress in nurses: a description of the risks for professionals. Texto Contexto Enferm. 2018;27(4):e4020017. DOI: https://doi.org/10.1590/ 0104-07072018004020017

23. Woods M, Rodgers V, Towers A, La Grow S. Researching moral distress among New Zealand nurses: a national survey. Nurs Ethics. 2015;22(1):117-30. DOI: 10.1177 / 0969733014542679

24. Wenwen Z, Xiaoyan W, Yufang Z, Lifeng C, Congcong $S$. Moral distress and its influencing factors: A cross-sectional study in China. Nurs Ethics. 2018;25(4):470-80. DOI: 10.1177 / 0969733016658792

25. Gomes D, Aparisi JCS. Deliberação coletiva: uma contribuição contemporânea da bioética brasileira para as práticas do SUS. Trab educ saúde. 2017;15(2):347-71. DOI: https://doi.org/ 10.1590/1981-7746-sol00052

26. Rodríguez Abrahantes TN, Rodríguez Abrahantes A. Dimensión ética del cuidado de enfermería. Rev Cubana Enferm [Internet] 2018 [cited 2020 Aug 21];34(3):749-60. Available from: http:// www.revenfermeria.sld.cu/index.php/enf/article/ view/2430

Recebido: 18 de janeiro de 2021 Aprovado: 1 de junho de 2021 Publicado: 14 de julho de 2021

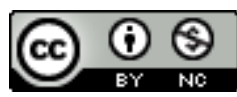

A Revista Baiana de Enfermagem utiliza a Licença Creative Commons - Atribuição-NãoComercial 4.0 Internacional. https://creativecommons.org/licenses/by-nc/4.0/ Este artigo é de acesso aberto distribuído sob os termos da Licença Creative Commons (CC BY-NC). Esta licença permite que outros remixem, adaptem e criem a partir do seu trabalho para fins não comerciais. Embora os novos trabalhos tenham de lhe atribuir o devido crédito e não possam ser usados para fins comerciais, os usuários não têm de licenciar esses trabalhos derivados sob os mesmos termos. 